\title{
A Discovery Scheme for Device-to-Device Communications in Synchronous Distributed Networks
}

\author{
Soojung Jung*, Sungcheol Chang* \\ *Electronics and Telecommunications Research Institute (ETRI), Korea \\ sjjung@etri.re.kr, scchang@etri.re.kr
}

\begin{abstract}
Device-to-Device (D2D) communications have been proposed as a means of realizing the potential advantage of the physical proximity of communicating devices, improving user experience and resource utilization. Discovery is one of the major design issues in the D2D communications, since they must discover each other and identify services provided by each other to directly communicate with one another. There are some requirements for discovery such as energy-efficiency (e.g. low duty cycle), scalability (e.g. support for high device density) and proximity-based autonomous detection in the D2D communications. In this paper, we propose a discovery scheme for D2D communications in synchronous distributed networks. In particular, we present a discovery scheme that each device advertises its presence and service and discovers other nearby devices autonomously and continuously, along with resource allocation in distributed manner. Using simulation, we evaluate the performances of our proposed scheme in terms of discovery latency and the number of discovered devices.
\end{abstract}

Keywords - D2D, Discovery, Synchronous distributed networks

\section{INTRODUCTION}

Device-to-Device (D2D) communication enables two devices in proximity of each other to communicate directly without going through the base station or access point. With increasing demand of high data rate wireless access for multimedia services, it has attracted much attention lately due to its potential for supporting proximity-based applications and services. D2D communication may either be networkcontrolled or may be managed by the devices without operator control. Recently, D2D communication in the networks through the cellular spectrum is being developed for LTEAdvanced in 3GPP [1]. It also has been developed for Wireless local-area network (WLAN) technologies based on the IEEE 802.11 standards (e.g. Wi-Fi Direct)[2] and is being developed for wireless personal-area-network (WPAN) technologies based on the IEEE 802.15 standards (e.g. Peer Aware Communication (PAC) in TG 8)[3] in the networks through the unlicensed spectrum.

For direct communication between devices, the discovery of neighbouring devices has been one of most important procedure since devices must discover each other and identify services provided by each other to directly communicate with one another. From [1] and [3], we can see that the following properties are desirable for discovery in D2D communication:

- Energy efficiency (e.g. low duty cycle)

- Proximity-based autonomous detection

- Expedited discovery

- Scalability (e.g. support for high device density)

In the networks in the unlicensed spectrum such as wireless sensor network or mobile ad-hoc network, the objective of discovery is only to find the neighboring devices to establish a good route to the sink at crisis applications (e.g. emergency/rescue operations). Therefore identifying neighboring devices in the sensor network is very important so that a device can get routing information about its surrounding, where no central controller can offer that information [4]. On the other hand, D2D communication in the above mentioned networks (i.e., LTE-Advanced, Wi-Fi Direct or PAC) is considered as a promising technology for supporting a multitude of use cases (e.g. local social networks) that have been achieved based on support of network servers via cellular networks [1], [3]. Therefore, based on signals communicated directly among device instead of the support of network server, a device has to identify whether neighboring devices can provide the set of available service or not. That is, there is a change of focus from device discovery toward both device discovery and service discovery.

Devices in the Wi-Fi Direct network can exchange queries to discover the set of available service before link establishment or group formation using CSMA/CA protocol. It is implemented by means of service discovery queries generated by a higher layer protocol which are transported at the link layer using Generic Advertisement Protocol (GAS) specified by $802.11 \mathrm{u}[2]$. Due to the asynchronous nature of Wi-Fi networks, the device is assumed to always monitor the channel so as not to miss the signals from other devices. In addition, due to device's mobility and the limited transmission range of $\mathrm{D} 2 \mathrm{D}$ communication links, the neighboring devices can vary over the time. To do deal this problem, continuous and autonomous discovery is needed. However, continuously trying to discover other devices may require an excessive power consumption to battery constrained devices. In addition, collisions in channel access increase rapidly as the number of contending devices increase. So the service discovery scheme 
based in the Wi-Fi Direct is not efficient in terms of energy consumption and resource utilization. On the other hand, if transmission and reception periods of certain signals are known to devices with a fixed frame structure, then various power management schemes can be implemented easily. A fixed frame structure is suitable for achieving high spectral efficiency and network throughput in a synchronous system. Discovery schemes in a synchronous system have also been proposed [5], [6].

There are two ways to perform discovery:

- Broadcast own information periodically without expectation of response from the receiver[5],[6]

- Exchange queries (i.e., request and response) [2]

Discovery based on Broadcasting can support autonomous and continuous discovery regardless of device's mobility. On the other hand, discovery based on queries can get responses from adequate devices quickly if there is no collision in resource access. In both [5] and [6], the way to perform discovery is broadcasting manner. However, often the device is looking for the device with known identity. Therefore, discovery based on queries is also considered for latency in a synchronous system. This paper describes a discovery scheme in the synchronous distributed networks to satisfy the above requirements for discovery.

The rest of this paper is organized as follows. In section II, we present the proposed discovery scheme, in particular structure of resource and construction of discovery signal. The overall simulation framework and results are presented in Section III to evaluate the proposed schemes. Finally, in section IV, we present conclusions.

\section{A DISCOVERY SCHEME IN THE SYNCHRONOUS DISTRIBUTED MANNER}

To satisfy the above requirements for discovery, the resource structure and resource selection manner of propose scheme are discussed. Then, the discovery signal of the proposed scheme is described. The major point of design is the integration of service discovery into device discovery at link layer (e.g., using messages generated in MAC layer) and the integration of discovery manner based on broadcasting manner and discovery manner based on queries. A possible scenario of application for the proposed scheme is the PAC system.

\section{A. Resource structure and resource selection for discovery}

We focus on a distributed discovery of D2D communication based on a synchronous OFDM-based physical layer where there is no central controller. Time Division Multiplexing (TDM) is used for partitioning channel resources and synchronous frame structure enables devices to operate at a very low duty cycle. Synchronization among all devices in the proximity is achieved in distributed manner. After synchronized, all the devices follow a hierarchical frame structure with fixed length, shown in Figure 1.

The frame structure, shown in Figure 1, is composed of frame, superframe and ultraframe. An ultraframe is consisted of multiple superframes. A superframe is consisted a number

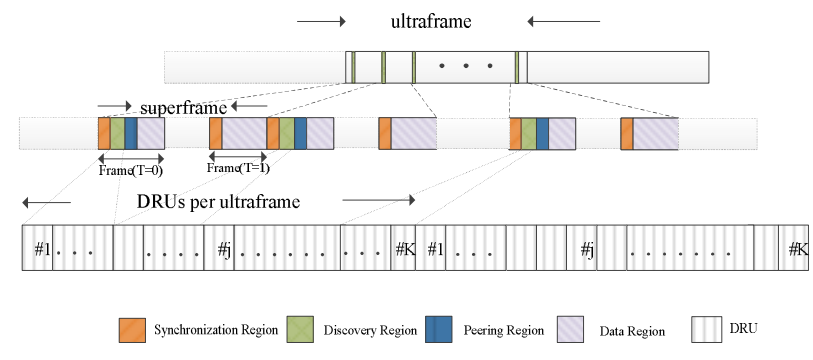

Figure 1. Resource structure for discovery

of frames. And there are two kinds of frame type. One is type 0 which includes regions for synchronization, discovery, peering and data. The other is Type 1 which only includes regions for synchronization and data. Peering is link establishment procedure between discovered devices for direct communication. And data traffics are transferred in the regions for data. A region for discovery is divided into a number of Discovery Resource Units (DRUs) which are used by devices to transmit or receive discovery signals. And there is the same number of DURs for each superframe. One discovery repetition period is an unltraframe. In one discovery repetition period, the discovery resource is divided into $K$ DRUs.

In both [5] and [6], the way to perform discovery is broadcasting manner where devices broadcast their discovery signals. However, how to assign and manage resources, are different. Each device randomly selects a resource for discovery signal in [5], but, each device select a resource which is the least congested from each device's respective in [6]. And in [6], it is shown that the resource selection manner called greedy selection outperforms the manner called random selection. Therefore, in this paper, a proposed scheme is based on the greedy selection to discover as many other devices as possible.

Each device picks a DRU from each device's respective when it powers up and transmits discovery signal including its identifier over that DRU. Each device also listens in the remaining DRUs to discover other devices in proximity. Exploiting the periodic nature of discovery signal for autonomous and continuous discovery, the devices can use the selected DRU in each repetition period without contention within a fixed frame structure as Figure 1.

However, due to the half-duplex constraint, devices cannot transmit and receive at the same time. Therefore, if multiple devices are transmitting signals over the same DRU, then a device cannot detect this collision during its transmission. To deal this problem, each device receives signals from other devices via the selected DRU without transmission of own discovery signal sometimes. If each device can detect signals over the selected DRU, it reselects a DRU based on same manner as previous one. In addition, to adapt the DRUs usage in the network to the changing network topology, reselections are also need.

\section{B. Construction of discovery signal}

To support multiple purpose and manner of discovery, the discovery signal of proposed scheme is generated in MAC 


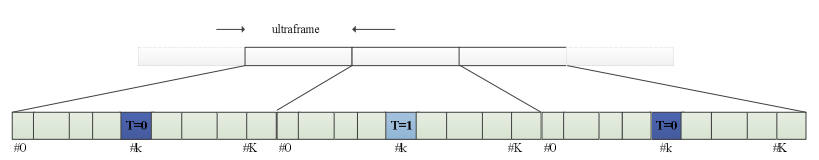

Figure 2. Example of supporting device discovery (type $=0$, device advertisement) and service discovery(type $=1$, service advertisement)

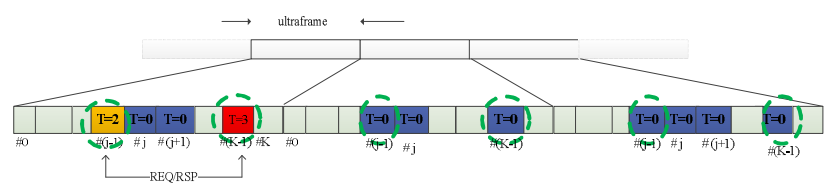

Figure 3. Example of supporting broadcasting (type $=0$, device advertisement) and Query(type $=2$ and type $=3$, service info request and service info response)

layer and includes type field representing whether the discovery signal is a signal for advertisement of own information for discovery or a signal for queries (i.e., request/response) related to service. It is including either own (or other) device identifier information (e.g., Device ID) or service information (e.g. application ID or user ID etc.) that the own (or other) device provides according to a value of the type field. In addition, Service Information Version (SIV) field in the discovery signal is used to represent the status of device's service configuration and to indicate changes in services configuration such as addition/deletion of applications and user change. By providing a parameter related to service information periodically (i.e., SIV with device discovery), exchange of queries for service discovery can be reduced. With these fields in the discovery signal, the proposed scheme can support both type of discovery manner (i.e., discovery based on broadcasting and discovery based on queries). And the proposed scheme can support not only device discovery but also service discovery at link layer without resource contention. For those, each device transmits its signal for device discovery with SIV over selected DRU periodically based on broadcasting in the proposed scheme. However, the contents of discovery signal may be changed according to its purpose. Example operations of proposed scheme are illustrated in the Figure 2 and Figure 3. The amount of time that a device spends to transmit each type of discovery signal is up to the implementation decisions (e.g. event-triggered or trade-off between discovery latency and energy savings etc.). Overall procedure of proposed scheme is illustrated in Figure 4.

\section{Simulation}

In this section, we present parameters and assumptions of system level simulation and numerical results for the proposed discovery scheme. We want to evaluate system performance of the proposed scheme and to get some insights about parameters (i.e. transmission range of reuse and number of DRUs) through simulations.

\section{A. Parameters and assumptions}

Performances of the proposed discovery scheme are evaluated through the system level simulations based on

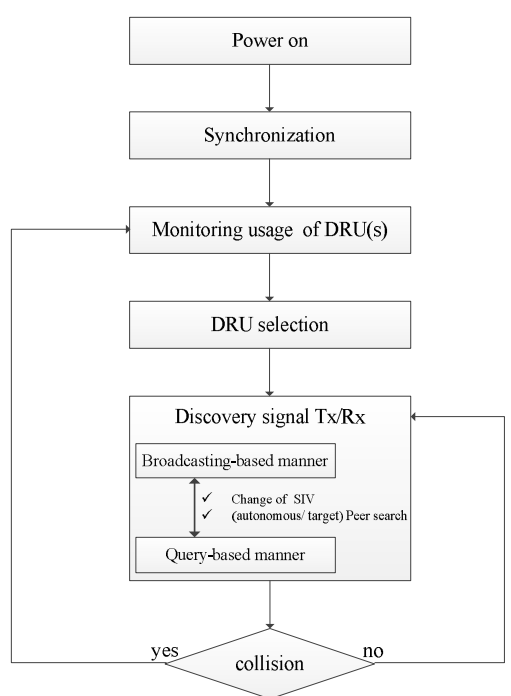

Figure 4. Procedure of propsoed scheme

parameters of IEEE 802.15.8[3]. Simulation Parameters for discovery are given in Table 1 .

TABLE 1. PARAMETERS AND ASSUMPTIONS

\begin{tabular}{|c|c|}
\hline Parameters & Value \\
\hline Max. Tx. Power $\left(P_{s}\right)$ & $20[\mathrm{dBm}]$ \\
\hline Tx./Rx. Antenna gain $\left(G_{t} / G_{r}\right)$ & $2.5[\mathrm{~dB}]$ \\
\hline Rx noise figure(NF) & $7[\mathrm{~dB}]$ \\
\hline Fade margin(M) & $8[\mathrm{~dB}]$ \\
\hline Noise density $\left(\mathrm{kT}_{0}\right)$ & $-174[\mathrm{dBm} / \mathrm{Hz}]$ \\
\hline $\mathrm{MCS}$ & BPSK, $1 / 2$ \\
\hline Bit rate( $(R)$ & $2.67[\mathrm{Mbps}]$ \\
\hline Bandwidth(B) & $20[\mathrm{MHz}]$ \\
\hline Device deployment & $\begin{array}{l}\text { Uniform random drip in } \\
D^{*} \mathrm{D} \mathrm{m}^{2}(\mathrm{D}=125 / 180 / 250)\end{array}$ \\
\hline Simulation time & $16[\mathrm{sec}]$ \\
\hline Discovery signal length & $128[$ bits $]$ \\
\hline $\begin{array}{c}\text { Path-loss at distance } d[\mathrm{~m}] \\
\text { between two devices }\end{array}$ & $L_{s}(d)[\mathrm{dB}]$ \\
\hline Frame length & $\begin{array}{l}\text { ultraframe }=0.8[\mathrm{sec}] \\
\text { superframe }=0.2[\mathrm{sec}], \\
\text { frame }=20[\mathrm{~ms}]\end{array}$ \\
\hline Total number of DRUs $(K)$ & $64 / 128 / 256$ \\
\hline Total number of devices $(N)$ & $64 \sim 1536$ \\
\hline
\end{tabular}

We assume that the $N$ devices are dropped in a square plane (D $\times \mathrm{D} \mathrm{m}^{2}$ area) with uniform distribution and $K$ DRUs available for discovery shown in Figure 5 and they are already synchronized. And we assume that devices join the network sequentially, i.e., a device selects the least congested DRU from each device's respective in a sequential manner. In 


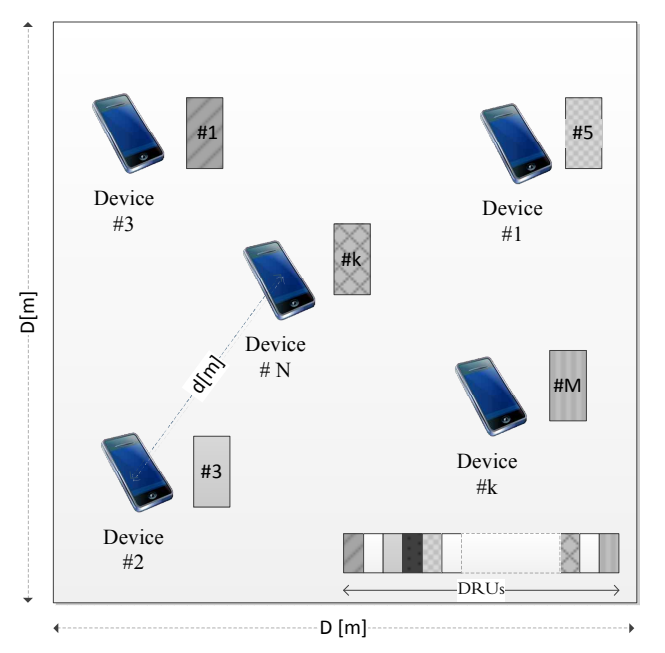

Figure 5. Deployment scenarios

addition, devices do not move around and no resource reselection manner is being used.

In order to obtain a performance model, we use a mapping function between the SINR and the PER and the curve is provided in [3]. The contents related to channel model are referred to [7] and only path-loss value for $2.4 \mathrm{GHz}$ band is considered. Interferences from devices collided in the same DRU are added as aggregate noise. The SINR of received discovery signal is obtained as follow:

$$
\begin{aligned}
& \frac{P_{S}}{P_{N^{\prime}}}=\frac{P_{s}}{P_{N}+P_{I}}=\frac{E_{b} R}{N_{0} B}=\frac{P_{t}^{\prime} G_{t}^{\prime} G_{r}^{\prime}}{P_{N} L_{s}^{\prime}(d) M^{\prime} N F^{\prime}+P_{I}} \\
& =\frac{P_{t}^{\prime} G_{t}^{\prime} G_{r}^{\prime}}{P_{N} L_{S}{ }^{\prime}(d) M^{\prime} N F^{\prime}+\sum_{i=1}^{N_{I}} \frac{P_{i}^{\prime} h_{i}}{L_{s}^{\prime}\left(d_{i}\right)}}
\end{aligned}
$$

where' denotes value in linear scale

\section{B. Simulation results}

The performance metrics are the average number of discovered devices, which is averaged over all devices through the network, and the CDF of "to discover" latency, which is the interval between the time that a device in receiving other device's signal is activated and the time that the device discovers successfully other device in transmitting discovery signal. The devices already discovered are not counted when those are re-discovered again.

We study multiple deployments with number of devices being different multiple of DRUs (e.g., from $1 \mathrm{x}$ to $12 \mathrm{x}$ ). The results are shown in Figure 6 and Figure 7. The average number of discovered devices according to the number of deployed devices is shown in Figure 6. And the average ratio of the number of total deployed device and the number of successfully discovered devices is shown in Figure 7. We can see that all devices discover each other as expected when number of devices is same as DRUs. However, at high densities all devices see a similar performance with each other discovering at most $50 \%$ even with $4 \mathrm{x}$ deployment density.

Next, we evaluate the effect of the number of DRUs (e.g., from 64 to 256) which is shown in Figure 8 and Figure 9. The

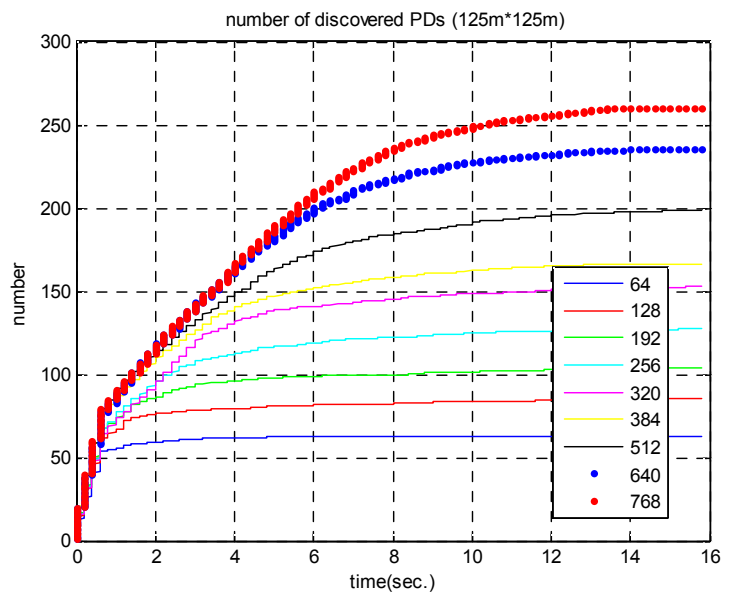

Figure 6. Average number of discovered devices with 64 DRUs

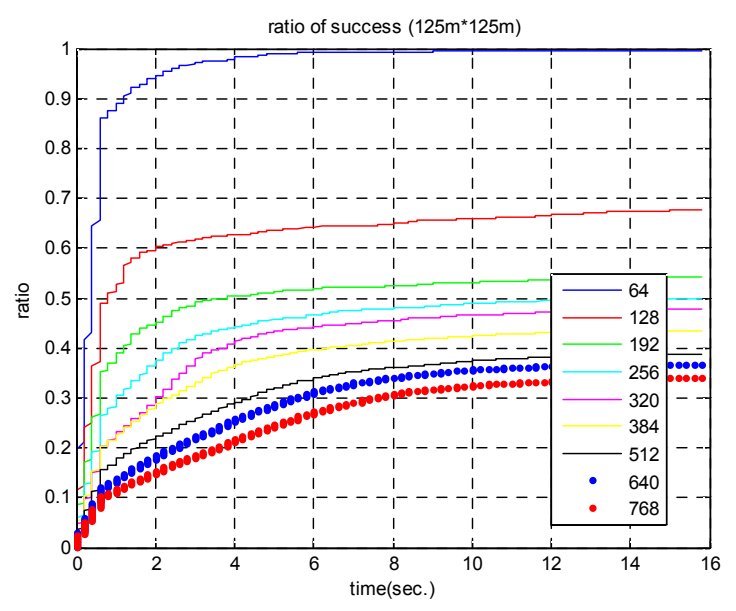

Figure 7. Ratio of success with 64 DRUs

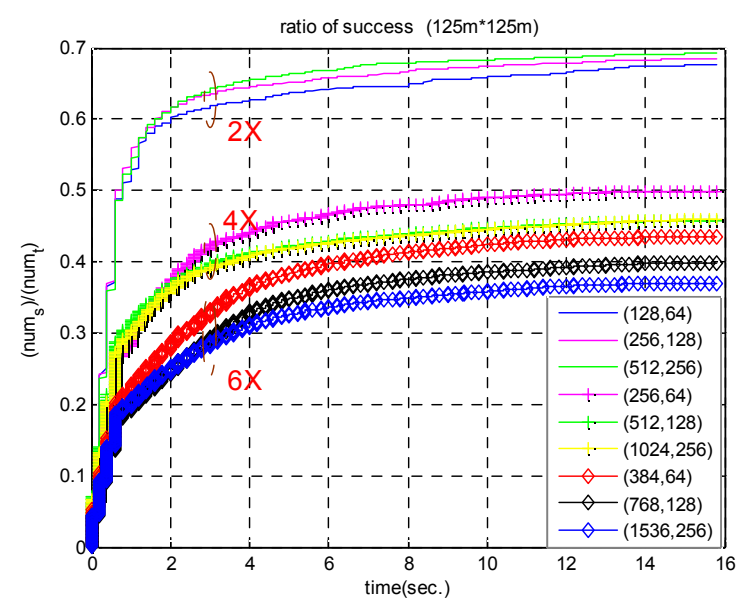

Figure 8. Ratio of success under various number of DRUs

total number of DRUs should be selected in order to occupy a small overhead of the system and to support dense environment. We can see that discovering performance is dependent on deployment density, not on the number DRUs in Figure 8 . Instead of the average number, the average ratio is 


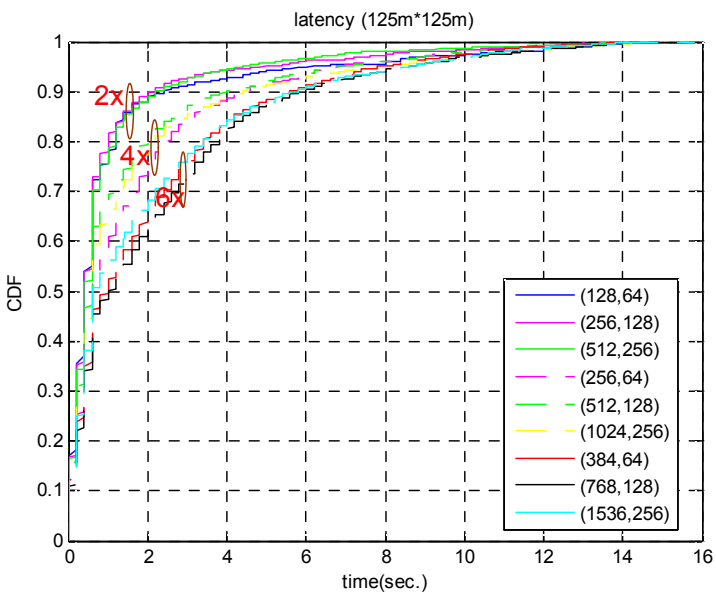

Figure 9. CDF of latency under various number of DURs

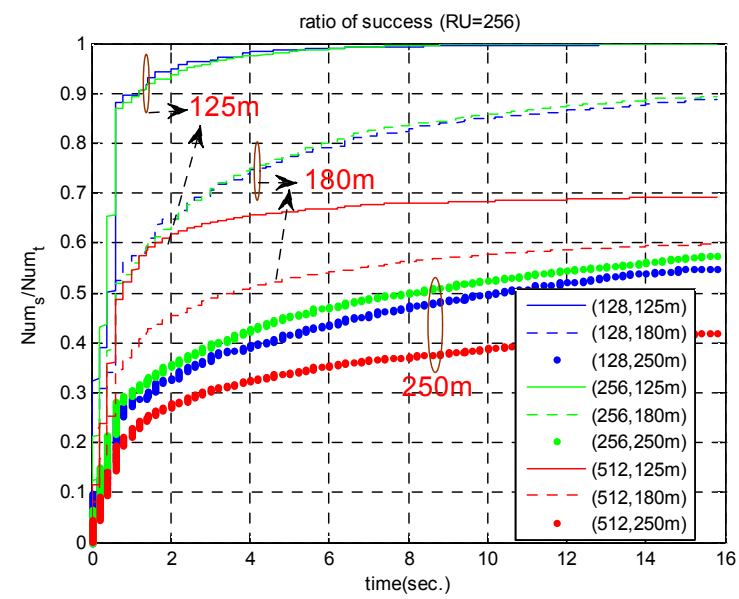

Figure 10.

Ratio of success under various transmission ranges

shown in Figure 8 . About $70 \%$ devices are discovered at $2 \mathrm{x}$ deployment density regardless of the total number of DRUs. In addition, from Figure 9, it is shown that latency is increased according to increase of deployment density, i.e., 2 seconds in $2 \mathrm{x}, 4$ seconds in $4 \mathrm{x}$ and 6 seconds in $6 \mathrm{x}$ for $90 \%$ of the discovered devices. So there is a trade-off between latency and system overhead devices. However, even though 256 DRUs, the overhead of proposed scheme is only $1.18 \%$ (i.e., $2.336 \mathrm{~ms}$ per superframe or $9.344 \mathrm{~ms}$ per ultraframe) Therefore, to support dense environment with adequate latency, it is desirable that the number of DRUs is chosen as the maximum value within a permitted range of system overhead. In case of 512 DRUs, it is expected that the proposed scheme can discover a thousand devices over in about 2 seconds with $2 \%$ overhead.

Finally, we evaluate transmission range for resource reuse which is shown in Figure 10. It is shown that all devices in $125 \times 125 \mathrm{~m}^{2}$ square planes with $2 \mathrm{x}$ deployment density and $90 \%$ devices in $180 \times 180 \mathrm{~m}^{2}$ square planes with $1 \mathrm{x}$ deployment density are discovered successfully. Therefore, we can see that the maximum transmission range for resource reuse exists between $60 \mathrm{~m}$ and $90 \mathrm{~m}$.

\section{IV.CONCLUSIONS}

In this paper, we proposed a discovery scheme in synchronous distributed networks to support device discovery and service discovery continuously and autonomously. Through system level simulations, we show that the proposed scheme is scalable for dense environment. It is also shown that specific value of range exists for frequency reuse and the number of DRUs to maximize the system performance.

\section{ACKNOWLEDGMENT}

This research was funded by the MSIP (Ministry of Science, ICT \& Future Planning), Korea in the ICT R\&D Program 2013.

\section{REFERENCES}

[1] G. Fodor et al., "Design Aspects of Network Assisted Device-toDevice Communications," IEEE Trans. Communications Magazine, vol.50, pp.170-177, 2012.

[2] D. Camps-Mur, A. Garcia-Saavedra and P. Serrano, "Device-to-Device communications with Wi-Fi Direct: overview and experimentation," IEEE Trans. Wireless Communications, vol.20, pp. 96-104, 2013.

[3] S.H. Park, B.J. Kwak, "TG8 Technical Guidance Document," IEEE 802.15-12-0568-07-0008, 2013.

[4] J.H. Jeon, A. Ephremides, "Neighbor discovery in a wireless sensor network: Multipacket reception capability and physical-layer signal processing," IEEE Trans. Communications and Networks, vol.14 pp.566-577, 2012

[5] A. Vigato, L. Vangelista, C. Measson, and X. Wu, "Joint discovery in synchronous wireless networks," IEEE Trans. Communications, vol 59, pp. 2296-2305, 2011.

[6] F. Baccelli et al., "On the design of device-to-device autonomous discovery," in Proc. Communication systems and Networks (COMSNETS), 2012.

[7] M. Hernandez, H.B. Li, I. Dotlićand R. Miura, "channel models for TG8," IEEE 802.15-12-0459-07-0008, 2012. 